EPJ Web of Conferences 66, 01003 (2014)

DOI: $10.1051 /$ epjconf/ 20146601003

(C) Owned by the authors, published by EDP Sciences, 2014

\title{
Many-body Quantum Reaction Dynamics near the Fusion Barrier
}

\author{
M. Dasgupta ${ }^{1, a}$, D.H. Luong ${ }^{1}$, D.J. Hinde ${ }^{1}$, and M. Evers ${ }^{1}$ \\ ${ }^{1}$ Department of Nuclear Physics, RSPE, Australian National University, ACT 0200, Australia
}

\begin{abstract}
The understanding of quantum effects in determining nuclear reaction outcomes is evolving as improved experimental techniques reveal new facets of interaction dynamics. Whilst the phenomenon of coupling-enhanced quantum tunnelling is understood to arise due to quantum superposition, the observed inhibition of fusion at energies well below the barrier is not yet quantitatively understood. Collisions involving weakly-bound nuclei, which have low energy thresholds against breakup, present further challenges. Recent coincidence measurements for reactions of weakly bound stable nuclei have not only provided a complete picture of the physical mechanisms triggering breakup, but have also shown how information on reaction dynamics occurring on time-scales of $\sim$ zepto-seconds can be obtained experimentally. These new experimental findings demand major developments in quantum models of near-barrier nuclear reactions.
\end{abstract}

\section{Introduction}

The quantum nature of many-body nuclear systems, and of their interactions, plays a dominant role in determining outcomes of nuclear collisions at energies near the fusion barrier. The fusion barrier results from a combination of the long-range repulsive Coulomb and the short-range attractive nuclear potential. Whilst it is natural to expect quantum effects in nuclear collisions, the recognition that these can dramatically change reaction outcomes arose from advances in accelerator technologies and experimental techniques [1]. Precision experiments in the last two decades [2-6], which went hand-inhand with theoretical developments, led to an understanding of the effect of quantum superpositions. Following a brief description of these advances, this paper discusses the current challenges faced in understanding the fundamental process of tunnelling of many-body nuclear systems, and the effects in reaction dynamics that are specific to the quantum structure of weakly bound nuclei.

\section{Effects of quantum superposition}

Experiments with heavy nuclei showed [7-10] that the measured fusion cross-sections at energies below the average fusion barrier can be orders of magnitude larger than the expectations of calculations assuming a single fusion barrier. The explanation of this phenomenon led to the realization that near-barrier collision dynamics is best described [11] by a linear superposition of the quantum states of the two interacting nuclei. A new picture of near-barrier reaction dynamics then emerged

\footnotetext{
a e-mail: mahananda.dasgupta@anu.edu.au
}

This is an Open Access article distributed under the terms of the Creative Commons Attribution License 2.0, which permits unrestricted use, distribution, and reproduction in any medium, provided the original work is properly cited. 
where the single fusion barrier can be thought to be effectively replaced by a distribution of barrier energies [11]. It was then proposed [12] that the barrier distribution can be obtained directly from experimental measurements. The experimental demonstration of the concept of the distribution of barriers however required precision measurements, which were pioneered by Jack Leigh at the Australian National University [2,3]. Results of two of these measurements for the fusion of ${ }^{16} \mathrm{O}$ with ${ }^{144} \mathrm{Sm}[5]$ and ${ }^{154} \mathrm{Sm}$ [2] are shown in Fig. 1. The plots show the second derivative of the fusion cross-section $(\sigma)$ multiplied by the centre-of-mass energy $(E)$ - a quantity which is effectively proportional to the probability of encountering a barrier of energy E. The dotted Gaussian curve shows the expected barrier distribution if only a single fusion barrier was involved; the smoothing arising from quantum tunnelling through a single barrier. It is clear that the measurements do not agree with the single barrier model, and are best described by the coupled channels calculations shown by the full lines. The vertical lines show the expected barrier energies using the eigenchannel approximation. The difference in the experimental barrier distribution presented in the left and right panels shows it depends on the nature of quantum states of the fusing nuclei. In reactions with ${ }^{144} \mathrm{Sm}$, the low-lying octupole vibrational state of ${ }^{144} \mathrm{Sm}$ (shown schematically in the top left corner of (a)) plays a major role, whilst for ${ }^{154} \mathrm{Sm}$, its low-lying rotational states play the major role (the level scheme is shown schematically in the left corner of (b)). The case of ${ }^{154} \mathrm{Sm}$, with low lying rotational states, allows a classical correspondence to be made; the ${ }^{154} \mathrm{Sm}$ nucleus can be pictured as a rugby ball shaped nucleus - the Coulomb barrier is lower when the spherical ${ }^{16} \mathrm{O}$ collides with the tip of the rugby ball, whilst a higher barrier arises for collisions on the side. A striking similarity can be found with experiments on collisions of ultra-cold ${ }^{40} \mathrm{~K}-{ }^{87} \mathrm{Rb}$ molecules [13], which have a permanent electric dipole moment. The collisions of these molecules proceed by means of tunnelling through a p-wave centrifugal barrier. When an electric field is applied the molecules align with the field - the attractive nature of the dipole-dipole interaction in the head-to-tail configuration lowers the barrier, while side to side collisions have an increased barrier. The modification to the height of the barrier changes the reaction rates - a situation similar to that in collisions with deformed nuclei $[2,3]$.

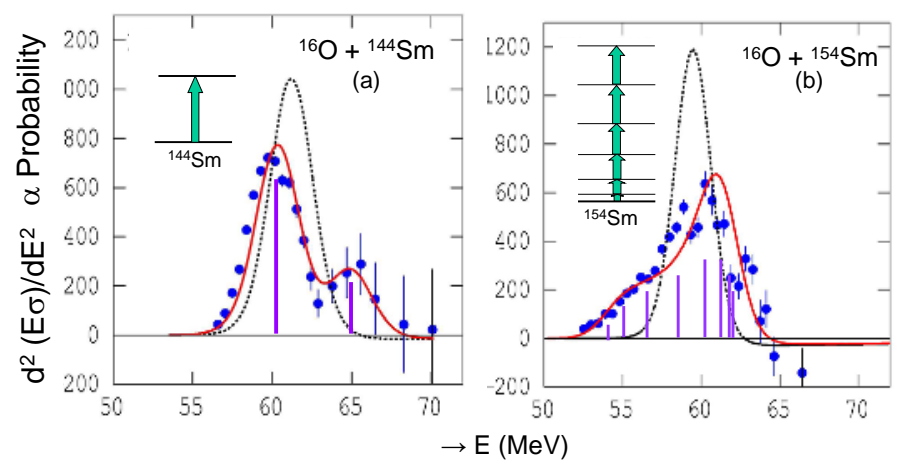

Figure 1. Experimental barrier distributions for ${ }^{16} \mathrm{O}$ fusing with ${ }^{144} \mathrm{Sm}$ (left panel) and ${ }^{154} \mathrm{Sm}$ (right panel) obtained from experiments which were amongst the first to make high precision measurement of fusion crosssections [2,5]. The quantity $d^{2}(E \sigma) / d E^{2}$ is proportional to the probability of encountering a barrier of energy $E$. The barrier distributions arise from a superposition of states. For ${ }^{144} \mathrm{Sm}$, coupling to the low lying octupole vibration state is the strongest, which effectively gives rise to the two-peaked structure seen in the barrier distribution. For ${ }^{154} \mathrm{Sm}$, the rotational states built on the ground state play the strongest role which give rise to a barrier distribution which is close to that expected classically from a prolate-shaped charged object. 
Effects of couplings to more complex states in nuclear collisions were observed in experiments led by Alberto Stefanini from Legnaro National Laboratory in Italy, where the effect of couplings to double phonon states and transfer were observed. The superposition effects change all collision outcomes such as elastic and inelastic scattering, transfer and fusion; however the effect on fusion [14] is most dramatic as fusion occurs via quantum tunnelling. The clear signatures of quantum superposition fundamentally changed long held ideas of fusion. The sensitivity of tunnelling probabilities to subtle changes in the potential, which is itself a construct to account for the many body interactions of protons and neutrons, indicates that fusion can be used to probe the underlying many-body quantum physics $[15,16]$. This goal links nuclear physics to other areas where understanding effects of quantum superposition and irreversible outcomes in complex quantum systems is a major research theme [17, 18], such as in atomic physics, photonics and biophysics [19].

\section{Reaction dynamics in collisions involving weakly bound nuclei}

Understanding the critical role in reaction dynamics of superpositions of states arose from studies of well bound nuclei [14]. For such nuclei the lifetimes of the low lying collective states are much longer than nuclear collision times of a few $10^{-22} \mathrm{sec}$. Does the reaction dynamics change when the lifetimes of low lying states are similar to or less than collision times? Answering this question is a challenge that is being addressed by performing experiments with weakly bound nuclei. It is indisputable that collisions of such nuclei must be described by a superposition of quantum states mentioned in Sec. 1. Understanding the role of low lying unbound states or short-lived resonances, which can lead to breakup of nuclei, is however a challenge [20-22]. Here the reaction dynamics becomes sensitive to both coupling effects and the effect of breakup [20-22]. One of the unambiguous observation of the consequences of the latter came through exploiting measurement of the fusion barrier distribution. Using beams of ${ }^{9} \mathrm{Be}$, it was shown unambigiously [23] that fusion cross-sections at above-barrier energies are suppressed compared with the expectations for well-bound nuclei. This general observation was later supported by theoretical calculations [24], and later this effect was seen in many other reactions using weakly bound stable [25-36] as well as unstable beams [22]. Answering whether fusion crosssections below the barrier are suppressed or enhanced compared with well-bound nuclei is however more fraught, since enhancement due to channel couplings and effects of breakup are both important. A clear answer requires model calculations and current models are not quantitative enough to obtain dependable predictions. Quantitative calculations firstly require a complete understanding and then modelling of the mechanisms leading to breakup. Understanding breakup mechanisms, particularly of $\alpha$-cluster nuclei, has had a long history. The most obvious breakup mechanism, i.e. resulting from excitation of states (or resonances) above the breakup threshold, is well documented [37, 38]. In addition to this mechanism, breakup following transfer of nucleons had also been observed [38, 39]. Such a mechanism is likely to be significant in low energy nuclear reactions involving halo nuclei in particular [40-42]. Breakup triggered by transfer has been shown to be important for weakly-bound stable beams. Recent experiments have provided a complete picture of all the physical mechanisms that trigger breakup [43-45], as well as important information on breakup time-scales. These experiments, described next, provide clear insights for incorporation into realistic models.

\section{Complexity of breakup of weakly bound nuclei}

Based on the cluster model picture of light nuclei, experimental studies had largely concentrated on investigating direct breakup into the initial cluster mass-partitions [46, 47], for example ${ }^{7} \mathrm{Li}$ breaking up into ${ }^{4} \mathrm{He}$ and ${ }^{3} \mathrm{H}$ nuclei. Whilst such breakup modes were observed, it was clear that more complex 


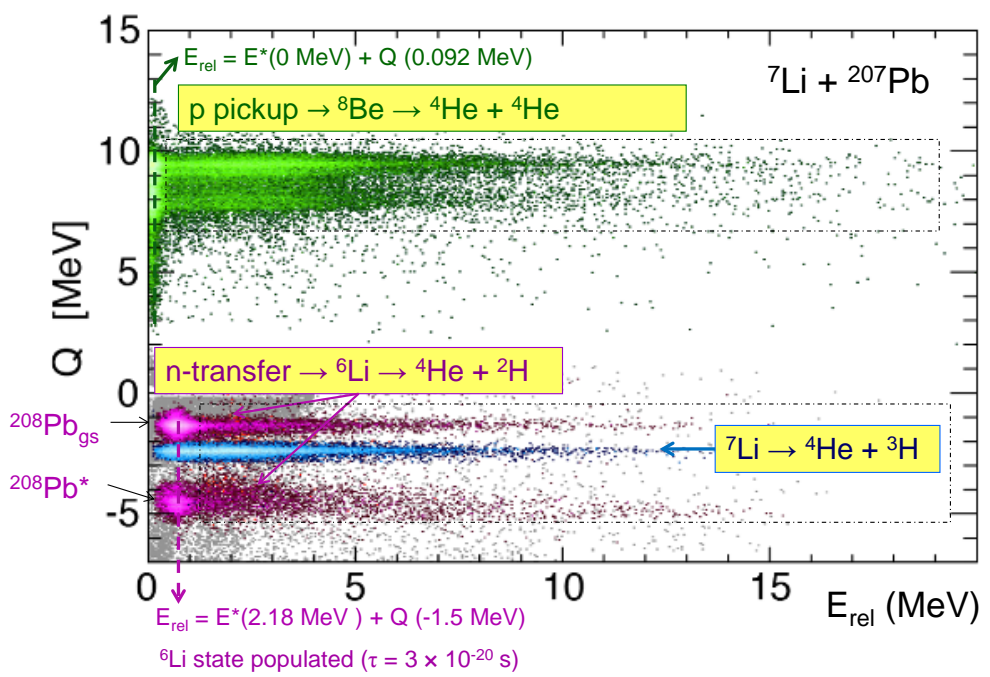

Figure 2. The reaction Q-value plotted against the relative energy of the two fragments $\left(E_{\text {rel }}\right)$ provides a complete picture of the breakup dynamics. Measurements are shown for a $29.0 \mathrm{MeV}$ beam of ${ }^{7} \mathrm{Li}$ incident on a ${ }^{207} \mathrm{~Pb}$ target. Coincidence pairs, $\alpha-d, \alpha-t$, and $\alpha-\alpha$ result from breakup following $n$-stripping, inelastic excitations and $p$-pickup, respectively. The measured $E_{\text {rel }}$ provides information on the state of the projectile-like nucleus prior to breakup and also gives information on breakup time-scales (see text). The dashed rectangles identify prompt breakup $\left(\sim 10^{-22} \mathrm{~s}\right.$ time-scale), whilst the peaks at small $E_{\text {rel }}$ (marked by vertical dashed lines) are due to breakup from long-lived states in the projectile-like nuclei.

mechanisms were also present [36, 39, 48-52]. Interpretations are not straightforward if experiments are done at energies above the barrier, as one or more of the breakup fragments can be absorbed leading to incomplete fusion. Interpretations are clearer if absorption is minimized by making measurements at energies below the barrier. Such experiments have been performed recently [44, 45] using beams of ${ }^{6,7} \mathrm{Li}$ from the Heavy Ion Accelerator Facility of the Australian National University (ANU). An array of four $400 \mu m$ thick double-sided silicon-strip detectors ${ }^{1}$ (DSSDs) was used to measure the coincident charged breakup fragments. One element consisted of two DSSDs placed back to back, forming a $\Delta E-E$ detector telescope for particle identification purposes [44]. The detectors were placed in a lamp-shade configuration in the backward hemisphere. The front face of each DSSD is divided into 16 arcs and the back face is divided into 8 sectors. The intersection of each arc and sector defines the position $(\theta, \phi)$ of each detected particle. The measured $\theta$ and $\phi$ of the two particles along with their kinetic energies $\left(E_{i}\right)$ allow kinematic reconstruction of events, and determination of the reaction $Q$-value. Knowledge of the $Q$-values provides information about states populated in the target-like nucleus, but not about the short-lived states of the projectile-like nucleus. However, at breakup, the energy that is available for sharing between the breakup fragments is the sum of the breakup $Q$-value $\left(Q_{B U}\right)$ and the excitation energy of the state from which breakup occurs $\left(E^{*}\right)$. The information on the state of the projectile-like nucleus prior to breakup is thus contained in the relative energy $\left(E_{r e l}\right)$ of the two breakup fragments.

The reaction $Q$-value combined with the relative energy of the two breakup fragments is a powerful tool to completely characterize the various breakup processes. This is illustrated next using the

\footnotetext{
${ }^{1}$ Manufactured by Micron Semiconductors Ltd., England
} 
example of the reaction of ${ }^{7} \mathrm{Li}$ with ${ }^{207} \mathrm{~Pb}$, for which the plot of $Q$-value against $E_{\text {rel }}$ is shown in Fig. 2. All the breakup modes identified in Fig. 2 are also observed in collisions of ${ }^{7} \mathrm{Li}$ with ${ }^{208} \mathrm{~Pb},{ }^{209} \mathrm{Bi}$ and ${ }^{144} \mathrm{Sm}$ nuclei. The $\alpha-t$ pairs result from breakup of ${ }^{7} \mathrm{Li}$; this observation matches the expectation that ${ }^{7} \mathrm{Li}$ breaks up into its cluster constituents. However, other breakup modes are very prominent. The $\alpha-\alpha$ pairs originate from breakup of the ${ }^{8} \mathrm{Be}$ nucleus which is formed when ${ }^{7} \mathrm{Li}$ picks up a proton from the target nucleus ${ }^{207} \mathrm{~Pb}$. The peak $E_{\text {rel }}=92 \mathrm{keV}$ (marked by a vertical dashed line) is due to breakup of the ${ }^{8} \mathrm{Be}$ ground state, which is long-lived, with a mean life of $\sim 10^{-16} \mathrm{~s}$. This mean life is much longer than the collision time and thus breakup of ${ }^{8} \mathrm{Be}$ from its ground state is expected to occur well after the ${ }^{8} \mathrm{Be}$ reaches its point of closest approach to the target, and thus well beyond the range of the nuclear-attraction between the colliding nuclei. The connection between $E_{\text {rel }}$ and time-scale of breakup is discussed further in the next section.

The $\alpha-d$ pairs are due to the breakup of ${ }^{6} \mathrm{Li}$ formed in $n$-stripping reactions, with the corresponding nucleus ${ }^{208} \mathrm{~Pb}$ being formed in its ground state and excited states identified by the $Q$-values in Fig. 2. Corresponding information about the state of the projectile-like partner can be obtained from the $E_{r e l}$ spectrum. The vertical dashed arrow in Fig. 2 marks the $E_{r e l}$ value of the majority of the $\alpha-d$ events, and corresponds to $E^{*}=2.186 \mathrm{MeV}$ [53] which matches the energy of the first excited state of ${ }^{6} \mathrm{Li}$. The width of this state is $24 \pm 2 \mathrm{keV}$ [53], corresponding to a mean life of $3 \times 10^{-20}$ $\mathrm{s}$. This mean life is also much longer than the collision time and as in the case of breakup from the ${ }^{8} \mathrm{Be}$ ground state, here too the breakup of ${ }^{6} \mathrm{Li}$ from the $2.186 \mathrm{MeV}$ state is expected to occur well after the ${ }^{6} \mathrm{Li}$ reaches its point of closest approach to the target. The breakup of this state is thus too slow to result in incomplete fusion (capture of either breakup fragments $\alpha$ or $d$ by the target-like nucleus) at above-barrier energies. The breakup processes that can result in incomplete fusion (and thus reduce complete fusion) thus must necessarily occur from states with much shorter lifetimes, which breakup prior to reaching the fusion barrier. Information on breakup time-scales is thus crucial in understanding the reaction dynamics.

\section{Reaction time-scales - a new window into reaction dynamics}

The principle of obtaining breakup time-scales can be understood from a simple classical picture of the collision, shown schematically in Fig. 3. The collision is at an energy below the fusion barrier so that the probability of capture of the projectile or of the breakup fragments is negligible. The projectile, represented by the unfilled circle in Fig. 3, is incident on the heavy target nucleus (dark circle); the light shading around the target nucleus represents the region where nucleon transfer can occur. Following inelastic scattering or transfer, if the projectile-like nucleus is excited to a short-lived state then its breakup will follow rapidly, while it is still close to the target-like nucleus (Fig. 3(a)). Being close to the target-like nucleus, the two positively charged breakup fragments (small circles marked with '+') can feel a strong Coulomb repulsion in different directions. This results in the two fragments obtaining a large relative velocity and thus a large $E_{r e l}$. If, on the other hand, the projectilelike fragment is excited to a long-lived state then breakup does not occur until the projectile is far from the target-like nucleus (Fig. 3(b)). The two fragments are now repelled from each other only by the Coulomb interaction between them, which results in a small $E_{\text {rel }}$, corresponding to the energy released in breakup. The relative energy of the fragments therefore provides a measure of whether breakup of the projectile-like nucleus occurs close to the target-like nucleus or much further away. Indeed, the measured spectrum presented in Fig. 2 show breakup events that have narrow peaks at low $E_{\text {rel }}$ values (indicated by the vertical lines) which must correspond to breakup far from the target-like nucleus (as in Fig. 3(b)) and those that extend to high $E_{\text {rel }}$ values (events within dashed rectangles) which are associated with breakup close to the target-like nucleus as depicted in Fig. 3(a). 


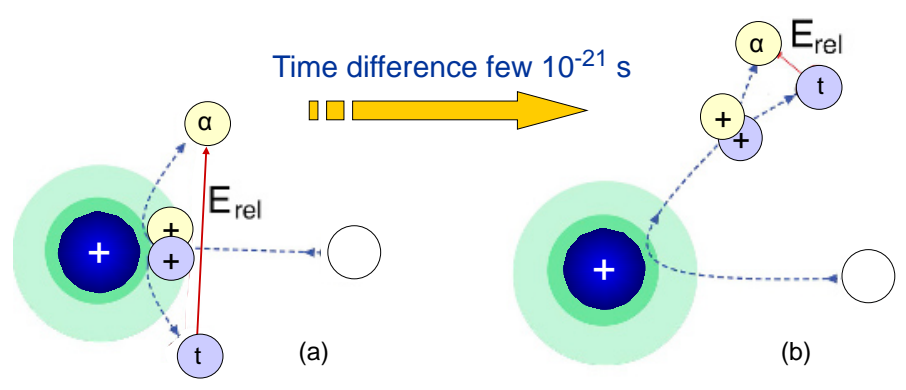

Figure 3. A schematic diagram of a collision leading to breakup at below barrier energies, pictured classically, showing different breakup scenarios and the effect on relative energy of the fragments $\left(E_{r e l}\right)$. The projectile incident from the right interacts with the target nucleus (dark circle); the light shading around the target nucleus indicates the interaction region. (a) If breakup occurs close to the target then the two fragments are repelled strongly giving rise to large $E_{r e l}$ values. (b) Breakup far from the target, results in smaller $E_{r e l}$ values. The $E_{r e l}$ values can thus provide a measure of the breakup time-scale.

The concepts outlined above can be put on firmer ground by making calculations of the most likely range of relative energies for the different breakup distances on the incoming and outgoing trajectories. The calculations were done using a three-body, three-dimensional classical trajectory model [54], which was developed to relate breakup and fusion. It has been applied successfully to explain the relationship between breakup and fusion of ${ }^{9} \mathrm{Be}$ on various heavy targets [43], and also in understanding the angular momentum distributions measured using isomer ratios [55] in incomplete fusion products following reactions of ${ }^{6,7} \mathrm{Li}$ with ${ }^{208} \mathrm{~Pb}$ and ${ }^{9} \mathrm{Be}$ with ${ }^{209} \mathrm{Bi}$. The calculations confirm the expectation that relative energy of the breakup fragments is a very sensitive measure of the breakup time-scale. Experimentally measured $E_{\text {rel }}$ can be thus be used to distinguish breakup from the two scenarios shown in Fig. 3(a) and (b), which have a time difference of a few $10^{-21} \mathrm{~s}$. If breakup of a nucleus occurs from a state with a life time greater than $\sim$ zepto-seconds, then breakup cannot affect fusion. Such breakup events are easily identified experimentally due to their narrow peaked distributions in $E_{r e l}$. Many previous studies have focussed on breakup from long-lived states. Although this can be a significant process, it cannot suppress complete fusion. The work presented here shows that to understand the effect of breakup on fusion, the focus must be on prompt breakup processes. For both the ${ }^{6} \mathrm{Li}$ and ${ }^{7} \mathrm{Li}$ induced reactions, prompt breakup following transfer is more likely than prompt direct breakup into the projectile cluster constituents. The transfer mechanism that triggers prompt breakup with ${ }^{6} \mathrm{Li}$ is $n$-stripping, whilst for ${ }^{7} \mathrm{Li}$ it is $p$-pickup. If prompt breakup occurs following transfer, then the lifetime of states of the projectile, and also of neighbouring nuclei that are populated following transfer, are important. This is a key insight to develop predictive models for use at the new accelerator facilities for unstable nuclei and their applications.

Thus far we discussed the role of timescales in processes prior to capture, which for lighter systems invariably leads to fusion. However in collisions of heavy nuclei, such as those used in forming heavy elements, capture does not necessarily lead to fusion. In such collisions, the di-nuclear system formed following capture can break apart due to the large Coulomb energy. The di-nucleus can break apart in less than $10^{-20} \mathrm{sec}$. An experimental measure of sticking time is useful for understanding the evolution of the di-nuclear system. The experimental method used for obtaining information on sticking times [56] has been discussed in the contribution by Hinde et al. in these proceedings. The measurements can distinguish between systems where the mean sticking time varies by a few $10^{-21}$ sec, which is ideal for probing fast dynamical processes such as quasi-fission. 


\section{Quantum tunnelling at energies well-below the barrier}

The realization of experimental probes of fusion barrier distributions, time scales in breakup and quasi-fission have all arisen from advances in experimental methods. Measurements of fusion can now be extended to energies well below the barrier, with cross-section measurements currently being obtained over a wider energy range than achievable in the first measurements. An example is given in Fig.4, which shows measurements of fusion cross-sections for ${ }^{16} \mathrm{O}+{ }^{208} \mathrm{~Pb}$, which span 8 orders of magnitude with very small uncertainties. As discussed in Sec. 1, at near barrier energies the outcomes are exquisitely sensitive to the effects of superposition; they are however not sensitive to processes that lead to the irreversible outcome of fusion inside the barrier. As the energy is lowered below the lowest barrier, the outcomes are sensitive to the processes at smaller separations as nuclei overlap. As nuclei overlap, the relative kinetic energy is converted to heating of the nuclei, which ultimately leads to the irreversible outcome of fusion - as envisaged by Neils Bohr's theory of formation of the compound nucleus. By choosing the energy we have very good control over the degree of overlap which allows us to investigate the gradual onset of irreversibility and also changes to the assumed inter-nuclear potential.

Experimental measurements have shown [57-61] that the tunnelling probability well below the lowest barrier is reduced compared to the expectations of the standard models of fusion that employ channel couplings and a Woods-Saxon shaped inter-nuclear potential. This effect, often referred to as fusion hindrance, is seen in a range of systems from fusion of isotopes of Nickel with Nickel [58] to even lighter nuclei [62] in pioneering experiments led by Cheng-lie Jiang from Argonne National Laboratory in the U.S.A. Understanding this suppression of tunnelling is currently a major challenge, and the consequences for reactions of astrophysical interests are currently being pursued by various groups. The theoretical explanations for reactions of heavier nuclei so far have centred around reduction of coupling strength as nuclei overlap [63] or by modification of the inter-nuclear potential, either due to incompressibility of nuclear matter [64] or due to a transition to an adiabatic potential [65]. However, it is problematic to obtain a reliable predictive model since the model parameters are often modified to fit the experimental observations.

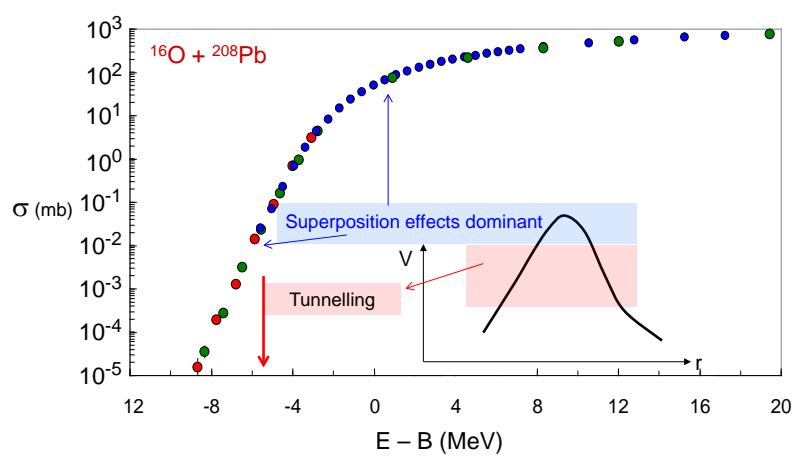

Figure 4. Measurements of fusion cross-sections for ${ }^{16} \mathrm{O}+{ }^{208} \mathrm{~Pb}$ made at energies well-below to well-above the average fusion barrier span 8 orders of magnitude with very small uncertainties. Measurements at energies close to the barrier are sensitive to quantum superposition effects, that lead to the observed distribution of barrier energies. Measurements at energies well below the barrier help understand quantum tunnelling of many body systems and together with measurements at energies well above the barrier they can be used to understand the onset of irreversibility in fusion. 


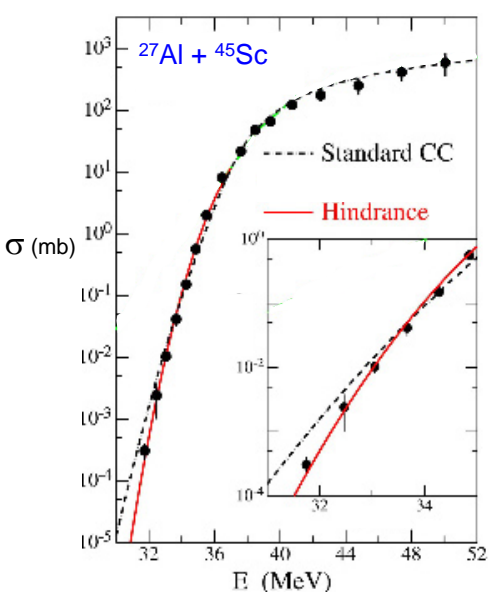

Figure 5. Cross-sections for fusion of ${ }^{27} \mathrm{Al}$ with ${ }^{45} \mathrm{Sc}$, measured by Jiang et al. at the Argonne National Laboratory, show the suppression of tunnelling when compared with the expectations of commonly used model of fusion. Understanding this phenomena, which is observed in many systems, is one of the current challenges.

Good agreement with the low-energy data effectively requires the potential barrier to be wider, and indeed the models that fit the data have a wider potential barrier - but is it really such a free parameter? Fortunately, the answer is no, since in a potential model both fusion above the barrier, which brings in higher angular momentum, and fusion below the barrier are equally affected at the same radial separation. Thus if the potential is changed to make calculated cross-sections agree with measurements then, in a potential model, fusion at above barrier energies is also changed. Indeed, the below barrier and above-barrier cross-section measurements for ${ }^{16} \mathrm{O}+{ }^{208} \mathrm{~Pb}$ cannot be explained by simply changing the potential [59] - if the potential is changed to fit the fusion well below the barrier, then the measurements at above barrier energies is not reproduced unless further dissipative effects are considered [66]. It is therefore clear that there is some fundamental understanding missing. The solution is likely to come from investigations of the sudden approximation sometimes made in calculating the potential, the reduction in coupling strengths to collective states with increasing overlap, and the transition from coherent superposition to irreversible outcomes in fusion, which is currently modelled as occurring abruptly well inside the barrier. Investigations of transfer reactions near- and below- the barrier indicate that interactions in the region where nuclei begin to overlap is playing a critical role in the reaction outcomes. At energies below the barrier, where suppression of tunnelling is observed, also observed are projectile like nuclei which are reflected back after transferring nucleons and suffering significant losses of kinetic energy [67]. One of the current areas of investigation is thus to understand the transfer of clusters of nucleons to high-lying excited states. A quantitative measure of such processes as a function of internuclear separation can serve as an input to model the onset of dissipation. One of the scenarios that is likely is that as the nuclei overlap, the loss of kinetic energy to internal excitations, which ultimately leads to the irreversible outcome of fusion is not a sharp transition as has been modeled traditionally. We need to realistically model the irreversibility to understand fusion of heavy nuclei. These experiments therefore link to one of the key challenges of modern physics, which is to understand how classical behaviour emerges in quantum systems due to interactions with an external environment. Nuclei are however completely isolated but the many complex excitations that occur as nuclei merge can be thought of as accessing an internal environ- 
ment. The standard ways to model interactions with an external environment do not seem applicable and new approaches to understand the tunnelling of complex objects is required.

\section{Outlook}

We are thus at an exciting time in the field, with once again theory and experiment going hand in hand, as was the case when coupling enhanced fusion was understood quantitatively. Research at energies near the barrier has thus been a fascinating area, which has brought to light quantum effects affecting reactions of many-body systems, which in the last twenty years has led to the understanding of coupling-assisted quantum tunnelling, and on this basis we are now moving forward in understanding in detail the emergence of the irreversible outcome of fusion.

The authors would like to thank Cheng-lie Jiang for sending us the results of his measurements. This work was supported by the Australian Research Council Discovery Grant DP110102858, and ARC Laureate Fellowship FL110100098 (MD).

\section{References}

[1] Treatise on heavy-ion science, See Volumes 1,2 and 7, edited by D. A. Bromley, Plenum Press, New York and London.

[2] J.X. Wei et al., Phys. Rev. Lett. 67, 3368 (1991).

[3] J.R. Leigh et al., Phys. Rev. C 52, 3151 (1995).

[4] A.M. Stefanini et al., Phys. Rev. Lett. 74, 864 (1995).

[5] C.R. Morton et al., Phys. Rev. Lett. 72, 4074 (1994).

[6] J.D. Bierman et al., Phys. Rev. Lett. 76, 1587 (1996); Phys. Rev. C 54, 3068 (1996).

[7] R.G. Stokstad et al., Phys. Rev. Lett. 41, 465 (1978); Phys. Rev. C 21, 2427 (1980).

[8] M. Beckerman et al., Phys. Rev. Lett. 45, 1472 (1980); Phys. Rev. C 23, 1581 (1981).

[9] S.G. Steadman, and M.J. Rhoades-Brown, Annu. Rev. Nucl. Part. Sci. 36, 649 (1986).

[10] M. Beckerman,Rep. Prog. Phys. 51, 1047 (1988).

[11] C.H. Dasso, S. Landowne, A. Winther, Nucl. Phys. A 405, 381 (1983); Nucl. Phys. A 407, 221 (1983).

[12] N. Rowley, G.R. Satchler, P.H. Stelson, Phys. Lett. B 254, 25 (1991).

[13] K.-K. Ni et al., Nature 464, 1324 (2010).

[14] M. Dasgupta, D.J. Hinde, N. Rowley, A.M. Stefanini, Annu. Rev. Nucl. Part. Sci. 48, 401 (1998).

[15] K. Hagino et al., Phys. Rev. Lett. 79, 2014 (1997).

[16] M. Dasgupta et. al., Phys. Rev. Lett. 99, 192701 (2007).

[17] W.H. Zurek, Rev. Mod. Phys. 75, 715 (2003); W.H. Zurek, Phys. Today 44, 36 (1991).

[18] M. Schlosshauer, Decoherence and the quantum to classical transition, Springer (2007).

[19] P. Ball, Nature 474, 272 (2011).

[20] J. F. Liang and C. Signorini, Int. J. Mod. Phys. E 14, 1121 (2005).

[21] L.F. Canto, P.R.S. Gomes, R. Donangelo, M.S. Hussien, Phys. Rep. 424, 1 (2006).

[22] N. Keeley, R. Raabe, N. Alamanos, J.L. Sida, Prog. Part. Nucl. Phys. 59, 579 (2007).

[23] M. Dasgupta et al., Phys. Rev. Lett. 82, 1395 (1999).

[24] K. Hagino, A. Vitturi, C. H. Dasso, and S. M. Lenzi, Phys. Rev. C 61, 037602 (2000).

[25] C. Signorini et al., Eur. Phys. J. A 5, 7 (1999). 
[26] M. Dasgupta et al., Phys. Rev. C 66, 041602 (2002).

[27] V. Tripathi et al., Phys. Rev. Lett. 88, 172701 (2002).

[28] Y. W. Wu et al., Phys. Rev. C 68, 044605 (2003).

[29] M. Dasgupta et al., Phys. Rev. C 70, 024606 (2004).

[30] A. Mukherjee et al., Phys. Lett. B 636, 91 (2006).

[31] P.R.S. Gomes et al., Phys. Rev. C 73, 064606 (2006).

[32] P.K. Rath et al., Phys. Rev. C 79, 051601 (2009).

[33] L. R. Gasques et al., Phys. Rev. C 79, 034605 (2009).

[34] M. Dasgupta, D. J. Hinde, S. L. Sheehy, and B. Bouriquet, Phys. Rev. C 81, 024608 (2010).

[35] C. S. Palshetkar et al., Phys. Rev. C 82, 044608 (2010).

[36] M.K. Pradhan et al., Phys. Rev. C 83,064606 (2011).

[37] O. Häusser et al., Phys. Lett. B 38, 75 (1972).

[38] K.O. Pfeiffer, E. Speth, K. Bethge, Nucl. Phys. A 206, 545 (1973).

[39] A. Shrivastava et al., Phys. Lett. B 633, 463 (2006).

[40] R. Raabe et al., Nature 431823 (2002).

[41] A. di Pietro et al., Phys. Rev. Lett 105022701 (2010).

[42] A. Lemasson et al., Phys. Rev. C 82044617 (2010).

[43] R. Rafiei et al., Phys. Rev. C 81, 024601 (2010).

[44] D.H. Luong et al., Phys. Lett. B 695, 105 (2011).

[45] D.H. Luong et al., Phys. Rev. C 88, 034609 (2013).

[46] N. Keeley et al., Phys. Rev. C 66, 044605 (2002).

[47] L. Fortunato and A. Vitturi, Eur. Phys. Jour. A 26, 33 (2005).

[48] C. Signorini et al., Phys. Rev. C 67, 044607 (2003).

[49] A. Pakou et al., Phys. Rev. C 76, 054601 (2007).

[50] D. Martinez Heimann et al., AIP Conf. Proc. 1098, 275 (2008).

[51] S. Santra et al., Phys. Lett. B 677, 139 (2009).

[52] F.A. Souza et al., Eur. Phys. J. A 44, 181 (2010).

[53] D. R. Tilley et al., Nucl. Phys. A 708, 3 (2002).

[54] A. Diaz-Torres et al., Phys. Rev. Lett. 98, 152701 (2007); A. Diaz-Torres, arXiv:0712.2275 (2007).

[55] L. R. Gasques et al., Phys. Rev. C 74, 064615 (2006).

[56] R. du Rietz et al., Phys. Rev. Lett. 106, 052701 (2011).

[57] C.L. Jiang et al., Phys. Rev. Lett. 89, 052701 (2002); C. L. Jiang et al., Phys. Rev. C 73, 014613 (2006).

[58] C.L. Jiang et al., Phys. Rev. Lett. 93, 012701 (2004).

[59] M. Dasgupta et al., Phys. Rev. Lett. 99, 192701 (2007).

[60] A. M. Stefanini et al., Phys. Rev. C 82, 014614 (2010).

[61] G. Montagnoli et al., Phys. Rev. C 85, 024607 (2012).

[62] C.L. Jiang et al., Phys. Rev. C 78, 017601 (2008).

[63] T. Ichikawa and K. Matsuyanagi, Phys. Rev. C 88, 011602(R) (2013).

[64] Ş. Mişicu and H. Esbensen, Phys. Rev. Lett. 96, 112701 (2006).

[65] T. Ichikawa, K. Hagino, A. Iwamoto, Phys. Rev. Lett. 103, 202701 (2009).

[66] H. Esbensen and Ş. Mişicu, Phys. Rev. C 76, 054609 (2007).

[67] M. Evers et al., Phys. Rev. C 84054614 (2011). 